complications were observed. We encountered in our early learning stage a failed attempted placement of Celt in one venous and one arterial access each $(0.98 \%)$ that remained asymptomatic. In one of these cases the Celt implant was accidentally deployed without properly attaching to the arterial wall. The device was dislodged into the popliteal artery without any flow restriction and the patient remained asymptomatic. Other adverse events were a complete and symptomatic occlusion of right external iliac artery, 1 patient presented with a delayed peripheral neuropathy. No early or delayed hematomas were observed whenever a proper device placement was achieved. On follow-up angiograms, several months following the placement, Celt implants were found in 18 cases adjacent to the arterial wall within the soft tissue. The extravascular migration remained asymptomatic and has previously been observed with other closure devices and may be related to arterial pulsation.

Conclusion Based on our experience Celt is very easy to use and an exceptionally safe and effective percutaneous closure device.

Disclosures W. Htun: None. N. Patel: None. R. Rabinovich: None. S. Newman: None. A. Wakhloo: None.

\section{E-122 THE FEASIBILITY AND EFFICACY OF TRANS-RADIAL ACCESS FOR MECHANICAL THROMBECTOMY IN ISCHEMIC STROKE: A SYSTEMATIC REVIEW OF THE LITERATURE}

A Kuczynski, M Goyal, M Almekhlafi*. University of Calgary, Calgary, AB, Canada

10.1136/neurintsurg-2019-SNIS.197

Background/Purpose The use of the radial artery to access the cervical and cerebral arteries is gaining popularity in the neuro-interventional field. This trans-radial approach (TRA) avoids the tortuosity that could be encountered in the aortic arch thereby shortening procedural times. This could be relevant in stroke endovascular thrombectomy procedures where time is critical. Large randomized controlled trials of coronary interventions have demonstrated that a TRA is associated with better outcomes and fewer access site complications than trans-femoral access (TFA). We conducted a systematic review to assess the safety and potential advantages of TRA for mechanical thrombectomy in adults with ischemic stroke.

Materials and methods We conducted a Medline search of the literature, including studies published in full in the English language with $\geq 5$ adult patients with acute ischemic stroke reporting on the procedures, success, and complications of TRA ( \pm TFA). Clinical and procedural variables were extracted and tabulated.

Results Sixty-eight studied were screened and five met our inclusion criteria. All studies were retrospective and conducted in the United States of America. A total of 73 patients with acute ischemic stroke underwent mechanical thrombectomy (median age 79 years, median initial NIHSS 18). In two studies, TFA was initially attempted but failed. Only one study included a TFA comparison group ( $\mathrm{n}=33$ patients). Mean access to reperfusion time was $76.3 \pm 36.0$ minutes (median 61.1 , range $35.8-132$ minutes) in TRA vs. 54.0 29.0 minutes (median 54.0, range 46-62 minutes) in TFA. Successful reperfusion (Thrombolysis in Cerebra Infarction score [TICI] $\geq 2 b$ ) was reported in $89 \%$ of the patients. Failure to reach the target occlusion was reported in $9 \%$ of TRA cases.
Conclusion TRA shows promising efficacy and efficiency for endovascular thrombectomy. Some of the available literature mostly reflects TRA use as a rescue access after failure to obtain TFA resulting in delaying reperfusion. Whether the routine use of TRA will result in comparable reperfusion rates to TFA and faster time to reperfusion is to be shown. Future studies on the TRA in stroke need to separately report the results of learning-phase cases and rescue TRA access.

Disclosures A. Kuczynski: None. M. Goyal: None. M. Almekhlafi: None.

\section{E-123 IMPROVED TICI GRADES IN PATIENTS WITH ACUTE LVO USING MECHANICAL THROMBECTOMY DUAL ASPIRATION TECHNIQUE FIRST 20 CASES}

J Brunson Jr*. Radiology, Bayonett Point Regional Medical Center, Hudson, FL

\subsection{6/neurintsurg-2019-SNIS.198}

Background and purpose Endovascular Mechanical Thrombectomy is the established standard of treatment for acute ischemic strokes for patients with large-vessel occlusions. Mechanical Thrombectomy techniques are well established in the literature which include many direct catheter aspirations and a choice of various stent retrievers or a combination of the above. Additionally some recent clinical studies demonstrate better procedural and clinical outcomes with balloon guide catheters vs other vascular accessories catheters.

Materials and methods We retrospectively compiled and reviewed the clinical and imaging outcomes of the last 20 consecutive patients who presented with acute intracranial LVO (January 2, 2019 - March 13, 2019) who were treated with emergent MT with concomitant stent retriever and'Dual Aspiration Technique'- (Penumbra and Stryker Aspiration at the level of both distal access catheter and carotid/vascular access sheath catheter).

Results Pt age range 59-105 years, average age-78.5 years, TICI 3 - 9 patients, TICI2 B - 10 Patients, TiCI 2A - 1, Successful recanalization rate $\%$ TICI $2 \mathrm{~B} / 3$ - 95\%. Average time to repefusion- 53.6 minutes. Failure rate- $0 \%$.

Conclusion Mechanical thrombectomy utilizing stent retriever and concomitant Dual Aspiration Technique appears to be feasible and effective for removal of thrombus in patients with AIS for LVO with high success rate of recanalization. Short/ midterm clinical data is however needed to for these patients. Disclosures J. Brunson: None.

\section{E-124 PERSISTENT TICI 0 AFTER MECHANICAL THROMBECTOMY: INCIDENCE AND INSIGHTS AT A HIGH-VOLUME COMPREHENSIVE STROKE CENTER}

${ }^{1} \mathrm{R}$ Mattay*, ${ }^{1} \mathrm{M}$ Cox, ${ }^{1} \mathrm{M}$ McCabe, ${ }^{1} \mathrm{~J}$ Schmitt, ${ }^{2} \mathrm{D}$ Kung, ${ }^{2} \mathrm{O}$ Choudhri, ${ }^{3} \mathrm{~B}$ Pukenas, ${ }^{3} \mathrm{R}$ Hurst. ${ }^{1}$ Radiology, Hospital of the University of Pennsylvania, Philadelphia, $P A ;{ }^{2}$ Neurosurgery and Radiology, Hospital of the University of Pennsylvania, Philadelphia, PA; ${ }^{3}$ Radiology and Neurosurgery, Hospital of the University of Pennsylvania, Philadelphia, PA

\subsection{6/neurintsurg-2019-SNIS.199}

Purpose There is now class 1a evidence for the efficacy of mechanical thrombectomy in patients with acute ischemic stroke and a large vessel occlusion (LVO). ${ }^{1}{ }^{2}$ Failure to recanalize portends a poor prognosis for the patient with a decreased chance for a good function outcome (modified 